\title{
Learners' perceived information overload in online learning via computer-mediated communication
}

\author{
Chun-Ying Chen ${ }^{\mathrm{a} *}$, Susan Pedersen ${ }^{\mathrm{b}}$ and Karen L. Murphy ${ }^{\mathrm{b}}$ \\ ${ }^{a}$ Center for General Education, National Taichung University of Education, Taiwan; \\ ${ }^{b}$ Department of Educational Psychology, Texas A\&M University, College Station, \\ Texas, USA
}

(Received 1 December 2010; final version received 8 April 2011)

\begin{abstract}
Many studies report information overload as one of the main problems that students encounter in online learning via computer-mediated communication. This study aimed to explore the sources of online students' information overload and offer suggestions for increasing students' cognitive resources for learning. Participants were 12 graduate students from two online courses in the United States. Their learning experiences in both online discussions and on the course website were explored through semi-structured interviews. They also completed a background questionnaire that assessed three constructs that limit learner readiness and are likely to lead to online students' perceived information overload: inadequate prior knowledge, inadequate English proficiency, and lack of technical skills for participating in computer-mediated communications. The findings suggest that varied learner characteristics led some students to be more susceptible than others to information overload. Emerging data-driven risk factors were: lack of efficiency in reading from computer screens, visual and auditory learning preferences, and time constraints. Difficulties associated with students' perceptions of information overload are addressed and implications for course design are offered.
\end{abstract}

Keywords: information overload; computer-mediated communication; cognitive load theory; online discussion

\section{Introduction}

With increases in Internet communication technologies, online learning has grown rapidly through the use of computer-mediated communication (CMC). CMC uses telecommunication technologies such as email, real-time chat, computer conferencing/online discussion systems, and online databases to support human communication between spatially separated learners (Jonassen et al. 1995). Although CMC can support teaching and learning by making information and communication easily accessible via computer networks, one of the main problems caused by the medium is information overload (IO) (Burge 1994; Eastmond 1995; Harasim 1987; Kear and Heap, 2007; Paulo 1999; Vonderwell and Zachariah 2005). IO becomes a problem when students simultaneously face having to acquire the technical skills necessary to participate in CMC and manage a large volume of information both on

*Corresponding author. Email: cychen666@gmail.com 
the course website and through computer conferencing (Harasim 1987). Numerous studies have investigated the problem of IO in the disciplines of organisation science, marketing, accounting, management information systems, business management, and psychology (for reviews, see Edmunds and Morris 2000; Eppler and Mengis, 2004; Klausegger, Sinkovics, and Zou 2007). Yet little research has been dedicated to investigating the problem of IO per se in online education as other disciplines have done. This study thus aimed to explore the sources of online students' IO and accordingly offer suggestions for increasing students' cognitive resources for learning.

\section{Theoretical framework}

\section{Cognitive load theory}

Cognitive load theory may provide a basis for understanding the concept of IO in this study. The theory consists of the cognitive architecture including memory and schema together with the information structures and their instructional implications that emphasise working memory constraints as determinants of instructional design effectiveness (Sweller, van Merriënboer, and Paas 1998). Knowledge is stored in long-term memory in the form of schema that acts as a single element. The sub-elements or lower-level schemas that are incorporated in the higher-level schemas no longer require working memory capacity. Understanding occurs when high-elementinteractivity material can be held simultaneously in working memory, and schema construction is the mechanism of this understanding process (Sweller, van Merriënboer, and Paas 1998).

Cognitive load can arise from three sources: intrinsic, extraneous, and germane cognitive load. Intrinsic cognitive load is intrinsic to the learning material, which has high element interactivity. Extraneous cognitive load is unnecessary load caused by inadequately designed instruction. Germane cognitive load comes from the effort that contributes to schema construction. While effective instructional design decreases extraneous cognitive load and increases germane cognitive load, increasing germane cognitive load will only work if the total load stays within working memory limits. Effective instructional techniques include the goal-free effect; the worked example effect; the completion problem effect; the split-attention effect; the modality effects; the redundancy effect; and the variability effect (for details, see Sweller, van Merriënboer, and Paas 1998). The first three instructional techniques are involved in problem-solving, in areas such as mathematics and science. The remaining four techniques are used in the presentation design of the learning material.

Valcke (2002) updated the current cognitive load theory by positioning prior knowledge and metacognitive load in the conceptual framework (Figure 1). Valcke stressed the contribution of prior knowledge in schema construction. Moreover, Valcke argued that learners invest effort in schema construction as well as in the monitoring of this activity, so the overall germane cognitive load is to be linked to the latter activity called metacognitive load.

\section{Information overload versus cognitive overload}

Memory and schema are key concepts of cognitive load theory that relate to the information-processing approach to learning (Valcke 2002). The human 


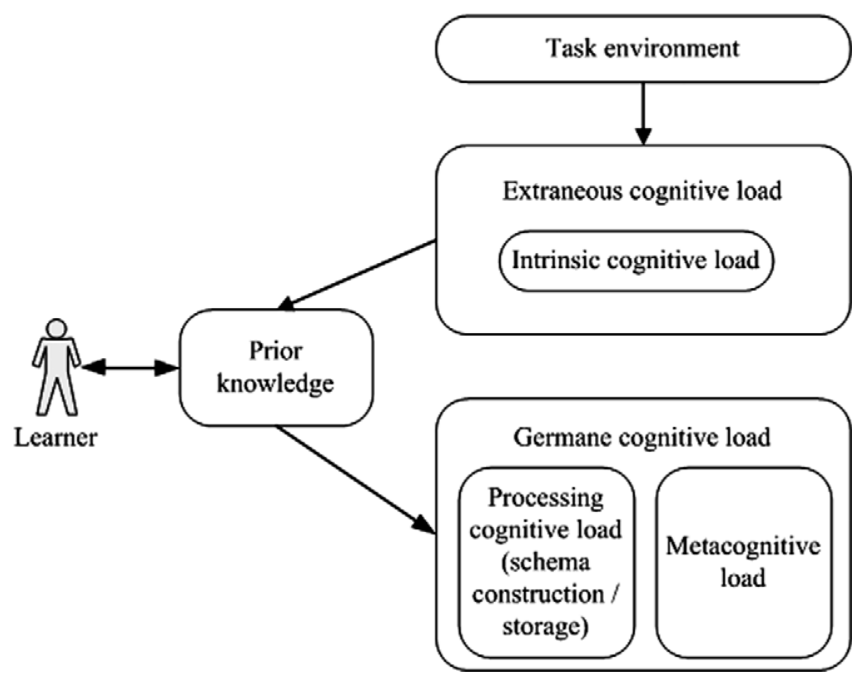

Figure 1. Valcke's (2002) updated model for cognitive load theory.

information-processing model thus provides a basis for understanding how IO and cognitive overload could possibly interfere with the cognitive processes (i.e., attention, storage, and retrieval) and metacognitive processes (that are responsible for guiding and monitoring the three cognitive processes) required for learning; that is, knowledge construction. Accordingly, the distinction between the two terms is illuminated.

As shown in Figure 2, IO and cognitive overload may occur in any of the cognitive processes as well as metacognitive processes to interfere with student learning. IO usually occurs in the attention process when an individual experiences any interference from the environment resulting in information loss, due to the limited

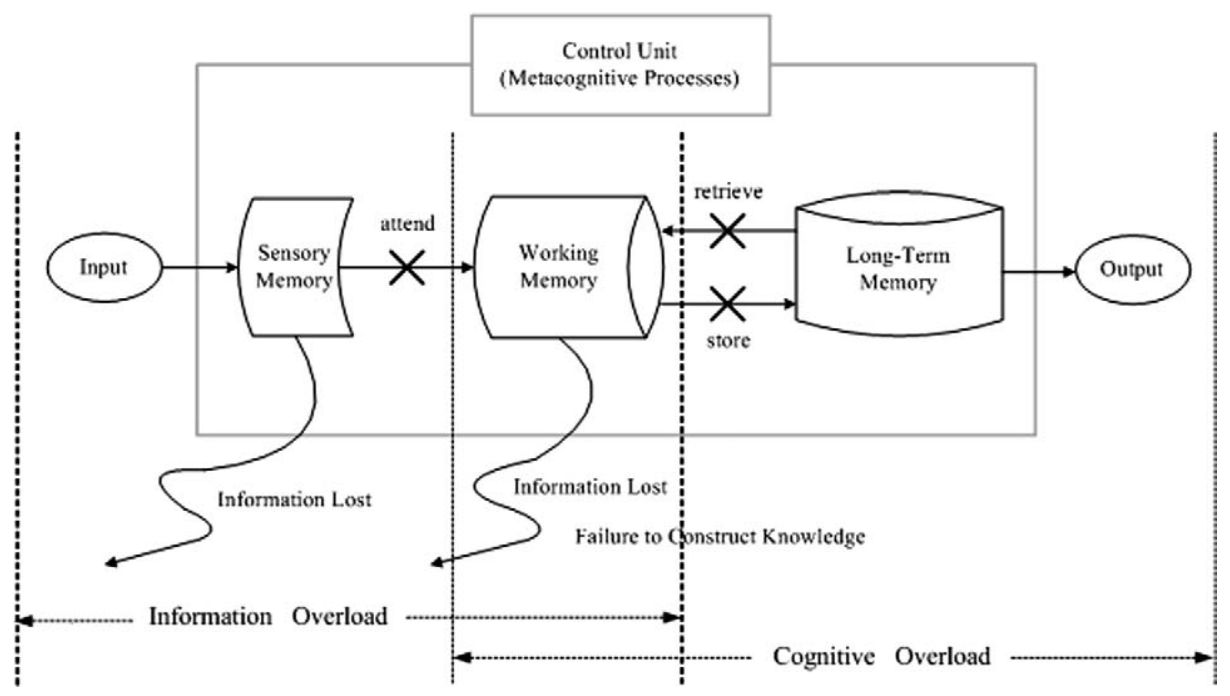

Figure 2. Information overload vs. cognitive overload. 
capacity of both the sensory memory and the working memory. Alternatively, cognitive overload happens in the storage and retrieval processes, resulting in the inability to connect new information to prior knowledge. When cognitive and metacognitive processes cannot function well for certain purposes, humans fail to construct knowledge. Thus IO seems to be the precursor of cognitive overload. There is an overlap between the two in that both emphasise limits in the capacity of working memory. The overlap may help clarify the common explanation that both terms refer to the same theory emphasising constraints on human working memory. Moreover, IO includes sensory memory that was omitted by cognitive load theory (Sweller, van Merriënboer, and Paas 1998; Valcke 2002).

In brief, cognitive overload is the load imposed on students during content learning, whereas IO is the 'noise' preventing students from learning content. IO caused by $\mathrm{CMC}$ in online learning is therefore in this study defined as the point at which a learner's capacity of sensory memory and working memory are exceeded, and the excessive information and stimuli from the CMC learning environment interfere with content learning. Thus, the concept of IO could be linked to the concept of extraneous cognitive load.

\section{Potential contributors to online students' perceived information overload}

Literature was reviewed to identify elements likely to contribute to online students' perceived IO, and revealed four dimensions of potential contributors: limited learner readiness, quantity of information, quality of information, and medium interface. First, three elements that limit learner readiness and are likely to lead to students' perceived IO are a lack of the technical skills for participating in CMC (Paulo 1999), inadequate prior knowledge (Valcke 2002), and inadequate English reading/ writing proficiency (Eastmond 1995). In CMC, students have to possess computer skills and computer conferencing skills in order to perform CMC tasks such as participating in online discussions and interacting online with others. Cognitive load theory emphasises the contribution of prior knowledge in schema construction that functions to reduce working memory load. Moreover, the ability to read and write well is necessary in the text-based CMC environment. Students with inadequate language proficiency may suffer reduced processing ability if the capacity in their working memory is exceeded by the tasks of reading and writing in English.

Second, three sources regarding quantitative components of information probably lead to students' perceived IO: large volumes of information from the Internet, and from the online discussion systems along with demands of course readings (Harasim 1987; Vonderwell and Zachariah 2005). In computer conferencing, active discussions generate heavy amounts of messages, and this problem increases with the group size.

Third, certain qualitative components of information help explain obstacles to processing information: difficult learning materials (Sweller 1994), text ambiguities (Trevino, Lengel, and Daft 1987), and redundant information (Mason and Kaye 1990; Schwan, Straub, and Hesse 2002). Difficult learning materials reflecting the inherent complexity of course content impose an intrinsic cognitive load (high element interactivity) on students. Sweller suggested that extraneous cognitive load that interferes with learning is a problem only under conditions of high cognitive load caused by high element interactivity. Text ambiguities cause students to misinterpret information. Text communication is a lean medium; it lacks the social cues (i.e. facial expressions, body language, and intonation) that aid participants' 
clarification and understanding of discussions. Students may experience text ambiguities while reading the learning material or conferencing messages. Redundant information refers to repetitive, irrelevant, or trivial information. Repetition of ideas may occur naturally due to the asynchronous attribute of the medium. For instance, while one is composing a message in computer conferencing, other students may be writing the same thing simultaneously (Burge 1994).

Last, interfaces of computer conferencing and online databases both can lead to learner disorientation and cognitive overload (Conklin 1987; DeStefano and LeFevre 2007; Hiltz and Turoff 1985). In computer conferencing, multiple simultaneous threaded discussions (a non-linear structure of discussions in which each topic is the starting point for a branch of responses and may be sorted by topic, date or author) can contribute to message fragmentation and be confusing to navigate and respond to. Further, multiple conference spaces can lead to disorientation. As for the hypertext interface of online databases, large numbers of links may overload and distract users with navigation and informational choices.

This study aimed to explore the sources of online students' IO and accordingly offer considerations for increasing students' cognitive resources to focus on content learning. Notice that we dealt with perceived IO rather than real IO because it is difficult to measure the real amount of information that an individual attends to and processes. Two questions helped to guide this research:

1. What learner characteristics cause students to be more likely at risk of IO?

2. What difficulties contributing to their perceptions of IO do students experience when they learn through the medium of CMC?

\section{Method}

\section{Online courses and participants}

Two online courses at a large university in the United States were selected purposefully because both: were a semester-long graduate-level course at a college of education; integrated computer conferencing as a component of course activities as a replacement for traditional classroom discussions; and required students to participate in online discussions. The instructional context of each class included a website and a computer conferencing system. The conferencing system offered the non-linear structure of threaded discussions. The learning activities required in the two courses were similar: both included small-group discussions, individual projects and critiques, and final papers.

Participants were 12 graduate students from two online courses in the United States. Of the 12 participants (five females and seven males), one-half were master's students and one-half were doctoral students. Five participants were full-time students, whereas seven were employed full-time. Students with different ranges of experience with technology use and online courses were selected purposefully.

\section{Data collection}

A mixed-method approach, with an emphasis on qualitative aspects, was chosen for the study to provide comprehensive descriptions of the phenomenon of IO in educational $\mathrm{CMC}$, and to enable cross-method triangulation. Two data sources were 
used in the study: semi-structured interviews and a background questionnaire. Interviews were used primarily to seek answers to both research questions; the quantitative data obtained from the questionnaire were used to support and verify the qualitative findings obtained in the interviews regarding research question one.

The literature review, as summarised in the earlier section 'Potential contributors to online students' perceived information overload', helped to inform the development of both the questionnaire and the interview protocol. The questionnaire was administered during the orientation meetings. Interviews were conducted during the fourth and fifth weeks of the semester because the literature indicated that IO is more pronounced early in a course, particularly during the first five weeks (Harasim 1987).

\section{Semi-structured interviews}

Interview questions (Table 1) were designed to examine the answers to research questions by exploring interviewees' learning experiences both in online discussions and on the course website. Additional probing questions were prepared to identify how the quantity of information, quality of information, and interfaces of CMC contributed to interviewees' perceptions of IO. The interviewees were guided to talk about their learning experiences in the online course they were taking. All interview

Table 1. Interview protocol.

Question

1. Tell me about your learning experiences in online discussions.

2. How do those difficulties influence your learning?

Probe:

Quantity of information

(a) How do you feel about the numbers of messages posted in online discussions?

Quality of information

(a) Do you ever encounter messages that you feel hard to understand?

(b) Do you ever encounter messages that seem ambiguous and that influence your learning? For example, in this sentence "Mary told Sue that she had won the beauty contest", it is not clear who won the beauty contest - Mary or Sue.

(c) Do you ever encounter messages that seem trivial and that influence your learning?

(d) Do you ever encounter messages that seem repetitive and that influence your learning? For example, someone may make the same or similar comments as others.

Medium interface

(a) How do you feel about the structure of how messages are arranged in online discussions?

(b) How do you feel about the multiple conference spaces in online discussions?

(c) Do you ever feel information is fragmentary and that it influences your learning?

3. Tell me about your learning experiences from the course website.

4. How do those difficulties influence your learning?

Probe:

Quantity of information

(a) How do you feel about the quantity of information on the course website?

Quality of information

(a) How do you feel about the structure of how information is arranged on the course website?

(b) Do you ever encounter information that you feel hard to understand?

Medium interface

(a) How do you feel about the number of links on the course website?

(b) Do you ever feel information is fragmentary and that it influences your learning? 
transcript analysis was consistent with the constant comparative method (Lincoln and Guba 1985). The constant comparative method is an inductive data analysis, which uses the specific raw data of transcripts to generate abstract categories. The analysis took the form of successive iterations involving the procedures based on Lincoln and Guba's techniques of unitisation and categorisation. The iterations were repeated until no new patterns emerged. Data collection and analysis continued until the categories were saturated (i.e. definitions of categories were well defined). To ensure credibility of the findings - that is, the trustworthiness of the data (Lincoln and Guba 1985) - extensive member checking with the interviewees was conducted throughout the study.

\section{Background questionnaire}

Three constructs that limit learner readiness and probably lead to online students' perceived IO were identified from the literature: inadequate prior knowledge, inadequate English proficiency, and lack of technical skills for participating in CMC. Accordingly, the background questionnaire used to identify students who were at risk of IO consisted of four parts: (I) prior knowledge, (II) English proficiency, (III) online course experience, and (IV) technology use experience.

Part I consisted of a series of questions (regarding students' undergraduate and/ or graduate major and the number of relevant courses taken) designed to determine participants' prior knowledge. Part II was a self-report survey that asked students to rank their English reading and writing proficiency as poor, fair, good, or fluent. Part III, an online course experience survey, inquired how many online courses participants had taken previously. Part IV was a technology use survey. This self-report survey asked participants to identify their level of skill on a scale ranging from one (low) to four (high) for a range of tasks including basic computer operation, file management, file transfer, email use, web browser operation, Internet use, computer conferencing, and information searching. This survey was adapted from Harvell's (2000) 'Background and Experience of Developers' questionnaire, which was adapted from the Bellingham Public Schools' (1999) Staff Use of Technology: 1999-2000 Self-Evaluation Rubric.

Table 2 provides criteria to identify interviewees' level of background knowledge/skills. The information provided in Parts I and II was used to identify each interviewee's level of prior knowledge and English proficiency. The results of Parts III and IV were combined to identify the level of technical skill required to participate in CMC. Accordingly, interviewees were identified as at risk of IO if they possessed a low level of any one of these variables: prior knowledge, English proficiency, or technical skill required to participate in $\mathrm{CMC}$.

\section{Results}

\section{Characteristics of learners susceptible to information overload}

Table 3 summarises interviewees' reported readiness for learning in the current online course based on data obtained from the questionnaire, and their perceived IO as revealed in interviews. This table shows that interviewees who were identified as at risk of IO usually did indeed express difficulties related to IO during the course. Participants were identified as at risk of IO if they possessed a low level of any one 
Table 2. Criteria to identify interviewees' level of background knowledge/skills.

\begin{tabular}{|c|c|c|c|}
\hline Part & Construct & Criteria & Level \\
\hline I & Prior knowledge & $\begin{array}{l}\text { Previous undergraduate or } \\
\text { graduate major was relevant } \\
\text { to that person's current major } \\
\text { Had taken at least one } \\
\text { course relevant to the } \\
\text { current course }\end{array}$ & $\begin{array}{l}\text { Low: satisfied } \\
\text { none } \\
\text { Medium: } \\
\text { satisfied either } \\
\text { one } \\
\text { High: satisfied } \\
\text { both }\end{array}$ \\
\hline II & English proficiency & $\begin{array}{l}\text { A native English speaker } \\
\text { Indicated either fluency } \\
\text { or possessing a good level of } \\
\text { English proficiency }\end{array}$ & $\begin{array}{l}\text { Low: satisfied } \\
\text { none } \\
\text { Medium: } \\
\text { satisfied either } \\
\text { one } \\
\text { High: satisfied } \\
\text { both }\end{array}$ \\
\hline III & $\begin{array}{l}\text { Online courses } \\
\text { experience }\end{array}$ & $\begin{array}{l}\text { Had not taken any online courses } \\
\text { previously } \\
\text { Had taken more than two online } \\
\text { courses previously }\end{array}$ & $\begin{array}{l}\text { Novice: } \\
\text { satisfied first } \\
\text { Moderate: } \\
\text { satisfied none } \\
\text { Experienced: } \\
\text { satisfied } \\
\text { second }\end{array}$ \\
\hline IV & $\begin{array}{l}\text { Technology use } \\
\text { experience }\end{array}$ & $\begin{array}{l}\text { Indicated an average level } \\
\text { of technology use that was: } \\
\text { - less than two } \\
\text { - two } \\
\text { - three or more }\end{array}$ & $\begin{array}{l}\text { Novice: } \\
\text { satisfied first } \\
\text { Moderate: } \\
\text { satisfied } \\
\text { second } \\
\text { Experienced: } \\
\text { satisfied third }\end{array}$ \\
\hline $\begin{array}{l}\text { III and IV } \\
\text { combined }\end{array}$ & $\begin{array}{l}\text { Technical skills for } \\
\text { participating in CMC }\end{array}$ & $\begin{array}{l}\text { Identified as a novice online learner } \\
\text { and technology user } \\
\text { Identified as a moderate/experienced } \\
\text { online learner and technology user }\end{array}$ & $\begin{array}{l}\text { Low: satisfied } \\
\text { first } \\
\text { Medium: } \\
\text { satisfied none } \\
\text { High: satisfied } \\
\text { second }\end{array}$ \\
\hline
\end{tabular}

of these variables: prior knowledge, English proficiency, or technical skill for participating in CMC. Survey findings thus identified six students at risk of IO. Additionally, each participant's reported difficulties related to IO were classified into one or more of these areas in terms of quantity of information, quality of information, and medium interface. Consequently, interview findings revealed that eight out of 12 students perceived IO. Two students (Alan and Carl) were identified as at no risk of IO in the survey, but their interviews revealed their perceived IO. In addition to the three constructs (as identified from the literature) leading students to be more susceptible to IO, other variables emerged from the interviews. The emerging data-driven variables were lack of efficiency in reading from computer screens, visual and 


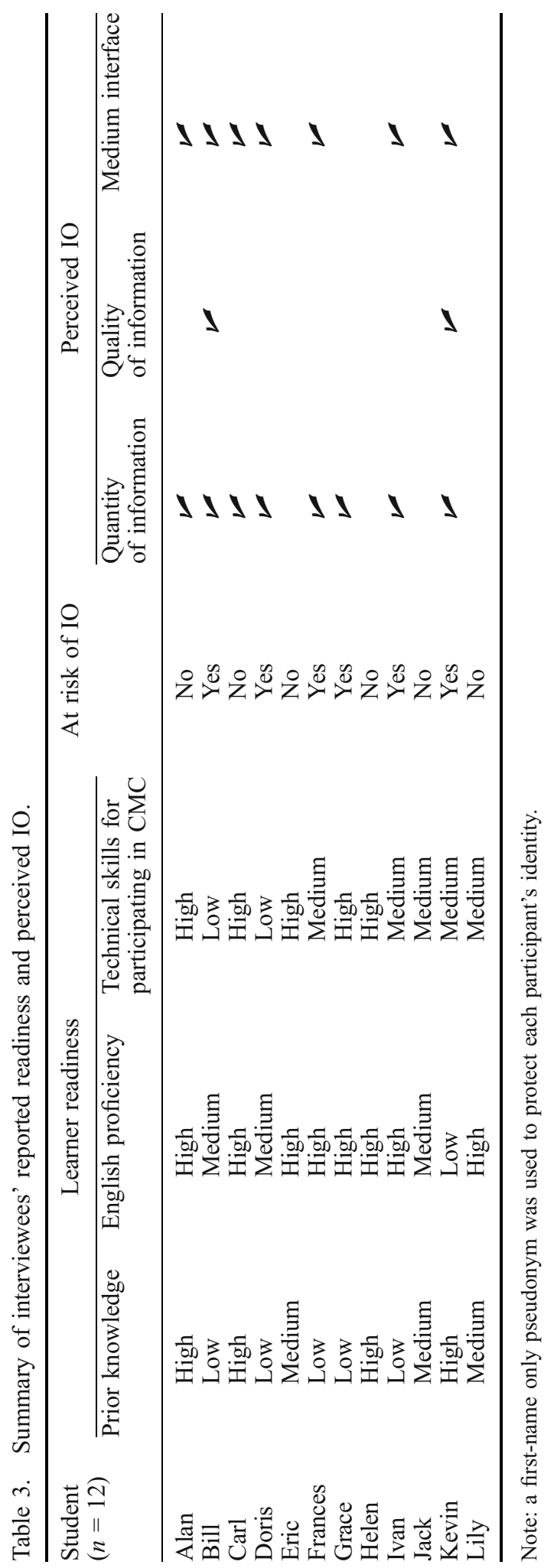


auditory learning preferences, and time constraints. Time constraints were mainly due to the demands of job and family responsibilities. IO was usually a result of several variables rather than a single one.

\section{Difficulties contributing to perceptions of information overload in CMC}

Interview findings revealed a number of difficulties that contributed to students' perceived IO, and were organised into six broad categories:

(a) connection problems;

(b) navigation difficulties;

(c) discomfort with online communication;

(d) demands of ongoing discussions and readings;

(e) difficulty in organising learning; and

(f) problems understanding the text-based readings.

\section{Connection problems}

Students who lacked technical skills for participating in CMC were observed likely to encounter connection problems. The students' connection problems were inability to $\log$ on to the conferencing system, and inability to access the electronic materials from the library. The first problem was usually due to the failure to set up the connection. The second problem was caused by a firewall. This firewall problem meant students were unable to access the electronic materials that were the required readings from the library. When they needed to pay attention to so much information at the beginning of the semester, students easily failed to attend to the connection instructions from the course website. Consequently, students might fall behind at the beginning of the semester. One student encountering the firewall problem was behind in coursework for approximately five weeks, as exhibited by participating late in each week's online discussion.

\section{Navigation difficulties}

Over one-half of the students, including experienced technology users, encountered navigation difficulties. Those difficulties included disorientation when browsing the web and computer conferencing, and difficulty linking discussion messages. First, students' disorientation on the web resulted primarily from external web resources, which were organised in a hypertext structure of more than three levels. Second, perceived disorientation in computer conferencing was due to the multiple conference rooms. When they were still unfamiliar with the conferencing structure and were unaware of their target location, some students became frustrated by spending a lot of time trying to find the target conference. Third, several students found it difficult to link discussion messages. Some students could not understand from the screen which person had replied to which message. One student offered a vivid metaphor to describe his confusion when the online discussions got busy: "It was like you sat in a room and talked to four or five persons at a time. Very confusing". Off-topic discussions in which someone changed the discussion topic without creating a new thread made navigation more difficult. 


\section{Discomfort with online communication}

Students' technical skills for participating in CMC and efficiency (speed and comprehension) in reading from computer screens influenced their level of comfort with online communication. Students who identified themselves as computer illiterate indicated that they needed to make a greater cognitive effort to figure out how to interact with the medium interface, while simultaneously having to process the discussion messages. Two students said that they had to print out all course materials because they had difficulty reading so much from a computer screen. One of these students said that his slow speed in reading from computer screens, his lack of computer skills, poor typing skills, and job-related and family-related time constraints made him feel reluctant to participate online. This led to infrequent logins and, subsequently, the number of his unread discussion messages mounted. Students reflected that online communication was a time-consuming process, so they appreciated the opportunities that classroom learning offered for immediate dialogue and immediate feedback loop.

\section{Demands of ongoing discussions and readings}

Over one-half of the students, especially those with insufficient prior knowledge, encountered this difficulty (i.e. numerous ongoing discussion messages along with the many resources provided on the course website). For students with full-time jobs and family responsibilities, this difficulty was a product of time constraints. However, some students finally developed information selection strategies to resolve the problem. They focused on information needed to fulfil course requirements and did not delve into extra resources unless time permitted.

\section{Difficulty in organising learning}

Over one-half of the students expressed difficulties in organising their learning. They indicated that organising learning was challenging when there were numerous ongoing learning activities, including continual online discussions. The following situations compounded this problem: demands of job, family responsibilities, or both; taking another online course at the same time; and novelty of the approach for first-time online learners who had to adjust to the differences of this mode of learning from the format of regular once-a-week on-site classroom learning.

\section{Problems understanding the text-based readings}

Students who identified themselves as slow readers and with visual and auditory learning preferences indicated that they had this problem. They could no longer rely on the instructor's lectures and verbal discussions for help as they had previously in classroom learning. This diagnosis is consistent with the self-report of their English proficiency obtained from the questionnaire, which revealed their English competence to be at only a fair level. The variables of both inadequate language proficiency and visual and auditory learning preferences evidently disadvantaged students in a text-based learning environment. 


\section{Discussion}

This study aimed to explore the sources of online students' IO and accordingly offer considerations for increasing students' cognitive resources to focus on content learning. Varied learner characteristics led some students to be more susceptible than others to IO. Those variables in turn probably led to students' difficulties in one or more of the following different dimensions: medium interface, quantity of information, and quality of information. Those difficulties, in turn, contributed to students' overall perceptions of IO. IO was usually a result of several variables rather than a single one. Moreover, over one-half of the students encountered both problems (i.e. demands of ongoing discussions/readings and difficulty in organising learning) in the dimension of quantity of information, and navigation difficulties in the dimension of medium interface.

The importance of prior knowledge was confirmed in this study. As indicated in Table 3, students with low levels of prior knowledge (i.e. Bill, Doris, Frances, Grace, and Ivan) were not only identified as at risk of IO but also revealed their learning difficulties associated with IO from their interviews. It was further observed that those students tended to encounter both problems in the dimension of quantity of information (i.e. demands of ongoing discussions/readings and difficulty in organising learning). High levels of prior knowledge imply that schemas are readily available in long-term memory and these schemas serve as advance organisers that help to interpret sensory information and link it to the existing schema (Valcke 2002). The available schemas thus provide executive guidance to the selection and processing of incoming information and thereby assist learners in their organisation of the learning activity (Kalyuga 2009).

It should be noted that some students with high levels of prior knowledge (i.e. Alan, Carl, and Kevin) also perceived IO, as revealed from their interviews. The variables that possibly led them to experience difficulties associated with IO included inadequate English proficiency, visual and auditory learning preferences, lack of efficiency in reading from computer screens, and time constraints. Cognitive load theory thus neglects characteristics of learners other than prior knowledge that should also be considered in certain learning contexts such as CMC. Rouet (2009) similarly emphasised the need to control other variables like individual characteristics and the task setting, not just learners' prior knowledge of the content area, in order to obtain reliable assessments of cognitive load and learning outcomes.

However, to deal with the problem regarding demands of ongoing discussions/ readings, several students relied on external means to identify relevant information to fulfil course requirements. The external means they mentioned included assignments, learning objectives, and overviews of the module content. Those external means were equivalent to the schemas that serve as advance organisers. The instructor can design such advance organisers or knowledge maps to provide students executive guidance as to what information to process, and how and in what sequence to process it.

Students identified as either novice online learners or having time constraints, including students with high prior knowledge, were observed to be more susceptible to the difficulty of organising learning. Those cases offer some considerations for online instructors. The literature noted that time management is an important factor in the success of most distance students, particularly those individuals in mid-life who are busy with the demands of job and family (Moore and Kearsley 1996). 
Such students need guidance to help them organise learning. Likewise, first-time online students need guidance in time management to adjust to the differences between online learning and on-site classroom learning. Online instructions that are designed and implemented from a constructivist perspective rely heavily on students to manage their own learning tasks and engage in interaction with peers and content (Vrasidas 2000).

Regarding the medium interface, the literature suggested consistently that students' navigation struggles are inevitable while browsing hypertext systems (DeStefano and LeFevre 2007). In the current study, students' reflections regarding the levels of interface layers may have implications for interface design. Two students who possessed a high level of computer competence indicated that, in their personal experience, people got confused easily when the hypertext structure was organised more than three levels deep. Similarly, other students remarked that they preferred a linear as opposed to a non-linear style for browsing web pages. When the number of interface layers exceeds three levels, users may feel that the information is fragmentary. The observations parallel the reports of earlier studies on hypertext environments that favoured linear presentation of material over hypertext formats because the linear formats provided more structure with less disorientation (Jonassen and Wang 1991; Nelson and Joyner 1990). Similarly, it has been reported that while navigating hypertext, students read the material in a more sequential manner and default to use of print-based text reading strategies to minimise cognitive load (Niederhauser et al. 2000).

The cognitive demands of the threading structure of computer conferencing, particularly the fragmentation of information caused by the non-linear discussion format, were evident to most students in both classes. Fragmented information creates a split-attention effect (Sweller, van Merriënboer, and Paas 1998) that may occur in computer conferencing when students have to integrate messages from different contributors regarding different topics. Off-topic discussions - when the line of discussion loses its coherence - create difficulties in linking messages belonging to a particular topic. In order to help students process online information more effectively, the instructor or discussion facilitators can offer guidance at the beginning of the course on how to navigate messages effectively by utilising system functions such as the selecting, sorting, and summarising features of computer conferencing systems. Several suggestions have been made to avoid fragmentary information and confusion in online discussions (Hewitt 2001; Salmon 2000).

\section{Implications for course design}

This study has several implications for course design. They consist of: readiness for online courses, design issues for the course website, and design issues for online discussions.

First, readiness for online courses is essential for reducing students' cognitive demands from learner-interface interaction (Hillman, Willis, and Gunawardena 1994), as well as ensuring their familiarity with course structure and expectations. The following suggestions will assist online educators in ensuring students' readiness:

- Most students in the present study indicated that the orientation sessions were an immense help in resolving technical problems, as well as offering an 
opportunity to understand the course requirements and the instructor's expectations. Thus, an orientation meeting may be the key to preparing students for the online course.

- An orientation session alone may not be sufficient to prepare students for learning online. Students may require more time to become familiar with the online environment. Two weeks is the period of time that most student participants in Conrad's (2002) study recommended for sufficient preparation.

- In order to give students enough time to resolve technological problems, the workload at the beginning of the semester should not be too demanding.

- It is important for the instructor to provide students with guidance in time management at the beginning; those with work or family responsibilities and novice online learners particularly need guidance to help them organise learning.

Second, this study suggests ways that the design of the course website might help manage IO:

- When having difficulties dealing with the quantity of information on the course website, several students noted that they relied on external means to identify relevant information to fulfil course requirements. The external means they mentioned included assignments, learning objectives, overviews of the module content, and fundamental concepts. The instructor can make use of external means to assist students with insufficient prior subject knowledge in identifying relevant information.

- Some students, particularly those learners with certain visual and auditory learning styles, commented that when they could not understand something on their own, they tried to interact with peers on the telephone, in person, or by email. Such learners can be encouraged to find learning partners. In addition, online examples of previous or current students' assignments would assist students' accomplishment of the learning tasks.

- To avoid navigation difficulties, most students in this study indicated that they preferred a linear as opposed to a non-linear style for browsing web pages. In addition, a web page containing more than three levels easily confused students.

Third, this study also suggests ways that the design of online discussions might help manage IO:

- First, the instructor or discussion facilitators can remind students how to navigate messages effectively when discussions become very active. Second, the instructor or discussion facilitators can weave and summarise messages at the appropriate time. Third, the instructor or discussion facilitators can reorient the discussions when they stray off-topic. Finally, the instructor or discussion facilitators can provide immediate guidance to students who are not on the right track.

- At the beginning of the course, the instructor or discussion facilitators can offer guidance and instructions on how to navigate messages effectively by utilising the system functions such as the selecting, sorting, and summarising features of the computer conferencing systems. 
- A deep level of non-linear style of conferencing structure is not preferred. Most students responded that when the levels of conferences went beyond three, they became easily confused.

\section{Conclusions}

It should be noted that the results of the current study are based on a small sample of student volunteers, and although quantitative data from a questionnaire were used, the emphasis is on qualitative findings. This study is limited to the context and setting of the two online courses, which needs to be taken into consideration to make transferability judgements.

Recommendations for further investigations follow. First, this study focused on a specific group of graduate students taking reading and writing intensive classes in the field of education. Similar studies could be carried out with different target learners such as undergraduate students, with learners majoring in different kinds of subject matter, or with students learning in different domains (e.g. scientific and technological fields). Second, this study examined a particular technology format (text-based $\mathrm{CMC}$ ). A similar study could be conducted in online courses incorporating different technologies such as audio, video, or multimedia technologies. Last, most research so far focuses on the external management of cognitive load by means of optimal instruction, but neglects consideration of learners' internal management strategies (Bannert 2002). In accordance with Bannert's call, future research may involve investigation on how learner characteristics and learning strategy use, particularly, metacognitive strategies, moderate perceived IO or cognitive load to achieve effective learning in different learning contexts.

\section{References}

Bannert, M. 2002. Managing cognitive load - Recent trends in cognitive load theory. Learning and Instruction 12: 139-46.

Bellingham Public Schools. 1999. Staff use of technology: 1999-2000 self-evaluation rubric. http://www.bham.wednet.edu/tcomp.htm.

Burge, E.J. 1994. Learning in computer conferenced contexts: The learners' perspective. Journal of Distance Education 9, no. 1: 19-43.

Conklin, J. 1987. Hypertext: An introduction and survey. IEEE Computer 20, no. 9: 17-41.

Conrad, D.L. 2002. Engagement, excitement, anxiety, and fear: Learners' experiences of starting an online course. American Journal of Distance Education 16, no. 4: 205-26.

DeStefano, D., and J.A. LeFevre. 2007. Cognitive load in hypertext reading: A review. Computers in Human Behavior 23: 1616-41.

Eastmond, D.V. 1995. Alone but together: Adult distance study through computer conferencing. Cresskill, NJ: Hampton.

Edmunds, A., and A. Morris. 2000. The problem of information overload in business organizations: A review of the literature. International Journal of Information Management 20: $17-28$.

Eppler, M.J., and J. Mengis. 2004. The concept of information overload: A review of literature from organization science, accounting, marketing, MIS, and related disciplines. The Information Society 20: 325-44.

Harasim, L.M. 1987. Teaching and learning on-line: Issues in computer-mediated graduate courses. Canadian Journal of Educational Communication 16, no. 2: 117-35.

Harvell, T. 2000. Costs and benefits of incorporating the Internet into the traditional classroom. Unpublished doctoral diss., Texas A\&M University, College Station, TX.

Hewitt, J. 2001. Beyond threaded discourse. International Journal of Educational Telecommunications 7, no. 3: 207-21. 
Hillman, D.C., D.J. Willis, and C.N. Gunawardena. 1994. Learner interface interaction in distance education: An extension of contemporary models and strategies for practitioners. American Journal of Distance Education 8, no. 2: 30-42.

Hiltz, S.R., and M. Turoff. 1985. Structuring computer-mediated communication systems to avoid information overload. Communications of the Association for Computing Machinery 28, no. 7: 680-9.

Jonassen, D., M. Davidson, M. Collins, J. Campbell, and B.B. Haag. 1995. Constructivism and computer-mediated communication in distance education. American Journal of Distance Education 9, no. 2: 7-36.

Jonassen, D.H., and S. Wang. 1991. Conveying structural knowledge in hypertext knowledge bases. Paper presented at the annual meeting of the Association for Educational Communications and Technology, February, in Orlando, FL.

Kalyuga, S. 2009. Knowledge elaboration: A cognitive load perspective. Learning and Instruction 19: 402-10.

Kear, K.L., and N.W. Heap. 2007. 'Sorting the wheat from the chaff': Investigating overload in educational discussion systems. Journal of Computer Assisted Learning 23: 235-47.

Klausegger, C., R.R. Sinkovics, and H. Zou. 2007. Information overload: A cross-national investigation of influence factors and effects. Marketing Intelligence and Planning 25, no. 7: 691-718.

Lincoln, Y.S., and E.G. Guba. 1985. Naturalistic inquiry. Newbury Park, CA: Sage.

Mason, R., and T. Kaye. 1990. Toward a new paradigm for distance education. In Online education: Perspectives on a new environment, ed. L. Harasim, 15-38. New York: Praeger.

Moore, M.G., and G. Kearsley. 1996. Distance education: A systems view. Belmont, CA: Wadsworth.

Nelson, W.A., and O.J. Joyner. 1990. Effects of document complexity and organization on learning from hypertext. Paper presented at the annual meeting of the Eastern Educational Research Association, February, in Clearwater, FL.

Niederhauser, D.S., R.E. Reynolds, D.J Salmen, and P. Skolmoski. 2000. The influence of cognitive load on learning from hypertext. Journal of Educational Computing Research 23, no. 3: 237-55.

Paulo, H.F. 1999. Information overload in computer-mediated communication and education: Is there really too much information? Implication for distance education. Unpublished master's thesis, University of Toronto, Canada.

Rouet, J.F. 2009. Managing cognitive load during document-based learning. Learning and Instruction 19: 445-50.

Salmon, G. 2000. E-moderating: The key to teaching and learning online. London: Kogan Page.

Schwan, S., D. Straub, and F.W. Hesse. 2002. Information management and learning in computer conferences: Coping with irrelevant and unconnected messages. Instructional Science 30: 269-89.

Sweller, J. 1994. Cognitive load theory, learning difficulty and instructional design. Learning and Instruction 4: 295-312.

Sweller, J., J.G. van Merriënboer, and F.G. Paas. 1998. Cognitive architecture and instructional design. Educational Psychology Review 10, no. 3: 251-96.

Trevino, L.K., R.H. Lengel, and R.L. Daft. 1987. Media symbolism, media richness, and media choice in organizations: A symbolic interactionist perspective. Communication Research 15, no. 5: 553-74.

Valcke, M. 2002. Cognitive load: Updating the theory? Learning and Instruction 12: 14754.

Vonderwell, S., and S. Zachariah. 2005. Factors that influence participation in online learning. Journal of Research on Technology in Education 38, no. 2: 213-30.

Vrasidas, C. 2000. Constructivism versus objectivism: Implications for interaction, course design, and evaluation in distance education. International Journal of Educational Telecommunications 6, no. 4: 339-61. 